\title{
ESTUDO DE CUSTO-RENDIMENTO DO PROCESSAMENTO DE QUEIJOS TIPO MINAS FRESCAL COM DERIVADO DE SOJA E DIFERENTES AGENTES COAGULANTES ${ }^{1}$
}

\author{
R. D. NEVES-SOUZA ${ }^{2, *}$, R.S. S. F. SILVA ${ }^{3}$
}

\section{RESUMO}

O desenvolvimento de alimentos é uma constante nas indústrias, nas universidades e nos institutos de pesquisa visando otimizar processamentos, agregar diferentes ingredientes em produtos já existentes, entre outros fatores. Após o desenvolvimento de um produto, informações adicionais podem ser obtidas pela determinação do rendimento e do custo do produto final. O objetivo deste trabalho foi estimar o rendimento e o custo de queijos Minas frescal tradicionais e adicionados de extrato hidrossolúvel de soja desengordurado (EHS) processados com coalho bovino e com coagulante microbiano (Aspergillus niger var. awamori). A introdução de $8 \%$ do EHS (em relação ao teor de sólidos totais do leite) no queijo Minas frescal processado com coalho bovino proporcionou rendimento em gramas de sólidos totais por litro de leite (g ST/L) superior de 16,67\% em relação ao queijo Minas frescal tradicional processado com o mesmo coalho. O queijo tipo Minas frescal processado com coagulante microbiano e com $8 \%$ de EHS apresentou rendimento (g ST/L) superior de 16,54\%, quando comparado ao seu tradicional. O custo das formulações-base (kg de queijo/100L de leite) processadas com coalho bovino e com coagulante microbiano foi US\$ 36,75 e US\$37,50, respectivamente, ao passo que os queijos tradicionais processados com os mesmos agentes coagulantes apresentaram custo equivalente a US\$35,12 e US\$35,36.

Palavras-chave: queijo Minas frescal; queijo com derivado de soja; quimosina produzida por fermentação; coagulante microbiano; extrato hidrossolúvel de soja.

\section{SUMMARY}

STUDY OF COST AND YIELD OF MINAS LIKE FRESH CHEESE PRODUCED WITH ADDED FAT FREE SOYBEAN HYDRO-SOLUBLE EXTRACT POWDER WITH CURD FORMED BY DIFFERENT COAGULANTS AGENTS. Food products development occur in industries, universities and research centers with the aim of optimizing processes, to include different ingredients to existing products or for other reasons. After developing a product, additional information may be obtained by determining yield and cost of the final product. The objective of this research was to estimate yield and cost of traditional Minas fresh cheese and those produced with added fat free soybean hydro-soluble extract powder (SEP), with curd formed by rennet or microbial coagulant (Aspergillus niger var. awamori). Minas fresh cheese with addition of $8 \%$ of SEP (in relation to total milk solids), processed with rennet had a yield (g TS/L) 16.67\% higher in comparison to the traditional cheese processed with the same rennet. The cheese processed with microbial coagulant and $8 \%$ of SEP had a yield (g TS/L) 16.54\% higher, when compared to the traditional cheese. The cost of the basic formulations with $8 \%$ of SEP (kg of cheese/100L of milk) processed with rennet or with microbial coagulant was US\$ 36.75 and US\$37.50 respectively, whilst that the traditional cheeses, processed with the same coagulants cost US\$ 35.12 and US\$ 35.36.

Keywords: Minas fresh cheese cheese with soybean derivative; chymosin produced by fermentation; microbial coagulant; hydrosoluble soybean extract.

\section{1 - INTRODUÇÃO}

A escolha do agente coagulante na produção de queijos é relevante porque ao lado de enzimas produzidas por bactérias, láticas ou não, as enzimas do agente coagulante influenciam na degradação protéica e umas são mais proteolíticas que outras [8]. A pepsina bovina, por exemplo, é uma das mais proteoliticas, é menos especifica que a quimosina e, pode hidrolisar excessivamente as caseinas, contribuindo com a diminuição do rendimento e promovendo sabor amargo ao produto, além de causar redução na vida-útil do queijo $[8,9]$.

Para minimizar esses problemas foi produzido um agente coagulante composto de $100 \%$ de quimosina utilizando-se das modernas técnicas de engenharia

1. Recebido para publicação em 23/03/2004. Aceito para publicação em 17/01/2005 (001314).

2. Cursos de graduação e pós-graduação na área de Nutrição da Universidade Norte do Paraná - UNOPAR, Av. Paris, 675, Jd. Piza, Londrina/ PR, CEP: 86041-100; e-mail: rejane.souza@unopar.br

3. Programa de Mestrado e Doutorado em Ciência de Alimentos da Universidade Estadual de Londrina - UEL.

* A quem a correspondência deve ser enviada. genética. Sua obtenção se dá pela fermentação de microrganismos transgênicos [3]. Este processo, conhecido como FPC (Fermentation Produced Chymosin) foi desenvolvido a partir de Aspergillus niger var. awamori. Inicialmente foi denominado de coagulante genético e recentemente passou a ser considerado como um coagulante microbiano, de acordo com informações do fabricante. Está sendo comercializado em diversos países pela Chr. Hansen com o nome de CHY-MAX [6]. Apesar das vantagens, especialmente no aumento do rendimento, a aplicação na indústria queijeira é pequena. O coalho bovino ainda é o mais utilizado no proces-samento de queijos, entretanto algumas pesquisas estão sendo desenvolvidas com o objetivo de comparar a influência dos diferentes tipos de agentes coagulantes, $3 / 4$ bovino e microbiano $3 / 4$ exercida sobre os parâmetros físico-químicos e sobre o rendimento de alguns tipos de queijos [3, 21], inclusive com o queijo Minas, um produto tipicamente nacional [23, 24, 25].

É o terceiro queijo mais fabricado no Brasil, em 2003 foram produzidas 31.762 toneladas $[1,5]$. Possui uma das mais simples tecnologia de fabricação, mesmo assim as técnicas industriais foram sofrendo modificações, ao longo dos anos, visando à melhoria de qualidade, o aumento no rendimento e à padronização do produto [4, 13, 14, 15, 19, 20, 22, 29, 30, 33, 34]. Além 
disso, tem sido estudada a introdução de sólidos de leite $[7,26]$ e de soja $[23,27,28,32]$ no processamento visando, principalmente, aumento no rendimento.

Diante disso, este trabalho teve como objetivo estimar o custo e o rendimento de queijos Minas frescal tradicionais e com extrato de soja desengordurado em pó processados com coalho bovino e com coagulante microbiano.

\section{2 - MATERIAIS E MÉTODOS}

\section{1 - Técnica de fabricação dos queijos}

O queijo Minas frescal, utilizado como referência, foi processado seguindo-se as recomendações de FURTADO \& LOURENÇO-NETO [12].

Foram utilizados oito litros de leite tipo C, 3/4 marca Tamalat, produzido pelo Lacticínio Tamarana Ltda. $3 / 4$, com teor médio de gordura de 3\%. Empregou-se coalho bovino em pó (Há-LA pó, Naturen) de acordo com as recomendações do fabricante. Adicionou-se cloreto de cálcio (Merk) a partir de uma solução aquosa (p/v), na proporção de $0,2 \mathrm{~g} / \mathrm{L}$ de leite, e $1 \%$ de cultura lática mesofilica, homofermentativa do tipo "O" (Chr. Hansen Indústria e Comércio Ltda.) em relação ao total de leite utilizado. O processo de salga foi realizado diretamente no leite, com o emprego de cloreto de sódio iodado (Cisne), na proporção de $1,5 \%$ em relação ao volume inicial de leite.

Os queijos tipo Minas frescal acrescidos de extrato hidrossolúvel de soja (EHS) desengordurado em pó (Olvebra Industrial S.A. de Porto Alegre/RS), baseouse na metodologia empregada por NEVES-SOUZA [23]. A quantidade de EHS empregada foi de $8 \%$ em relação ao teor de sólidos totais do leite. Uma formulação-base foi processada com $8 \mathrm{~L}$ de leite; $452 \mathrm{mg}$ de coalho bovino, $75 \mathrm{~g}$ de EHS, 4,0g de cloreto de cálcio, $120 \mathrm{~g}$ de cloreto de sódio e $8 \mathrm{~mL}$ de cultura lática. A única diferença que houve no desenvolvimento da formulação-base alternativa foi a substituição do coalho bovino por $1,9 \mathrm{~mL}$ de coagulante microbiano. Cada tratamento foi realizado em triplicata.

\section{2 - Composição centesimal}

Os teores de umidade, proteínas totais, gordura, cinzas totais e carboidratos totais do leite, da mistura (leite + EHS), dos queijos tradicionais, dos queijos acrescidos de EHS processados com coalho bovino e com coagulante microbiano, e dos respectivos soros, que foram obtidos a partir de cada um destes queijos, foram determinados seguindo-se a metodologia oficial da A.O.A.C. [2]. Estes dados foram utilizados para estimar as cifras de transição (item 2.3).

\section{3 - Cifras de transição}

As cifras de transição fornecem uma idéia da retenção dos componentes do leite no queijo e informam as perdas que ocorreram no soro, permitindo assim prever o rendimento de fabricação, independente de haver diferença na composição do leite utilizado, além destes dados se tornarem passiveis de uso em qualquer lugar, uma vez que sempre expressarão o que acontece com cada elemento do leite [13].

Para estabelecer as cifras de transição da gordura, da proteína e dos sólidos totais do leite e da mistura (leite + EHS) para os respectivos queijos foram consideradas as composições centesimais do leite e da mistura, bem como as perdas de cada constituinte pelo soro (item 2.2), seguindo as quantificações citadas por FURTADO \& WOLFSCHOON-POMBO [13].

\section{4 - Rendimento}

O rendimento de cada queijo (tradicionais e com EHS) foi estimado de duas maneiras:

a) em litros de leite necessários para a elaboração de um quilo de queijo (L/ kg). Neste caso, dividiu-se o volume de leite empregado pela soma da massa dos queijos obtidos [24];

b) em gramas de sólidos totais de queijo por litro de leite (g ST/L), conforme SABOYA et al. [26]. Para isto empregou-se a equação 1 .

$\mathrm{R}(\mathrm{g} \mathrm{ST} / \mathrm{L})=\mathrm{P} \times \mathrm{ST} \times 1$

\section{$\mathrm{V}$}

$\mathrm{R}=$ rendimento; $\mathrm{P}=$ quilos de queijos obtidos;

$\mathrm{ST}=$ percentagem de extrato seco dos queijos; $\mathrm{V}=$ volume de leite utilizado.

\section{5 - Custo dos queijos}

Estimou-se o custo dos queijos Minas frescal tradicionais e de cada formulação-base por meio da identificação das quantidades empregadas de matérias-primas (leite e derivado de soja), e coadjuvantes tecnológicos (agentes coagulantes, cloreto de cálcio, cloreto de sódio e cultura lática), em unidades coerentes de massa, necessários para cada tratamento. A partir destes valores calculou-se o custo para processar $100 \mathrm{~L}$ de leite. Os resultados foram expressos em dólares americanos.

\section{6 - Análise estatistica}

O teste de Tukey foi empregado para comparar as médias obtidas a partir de cada análise e pelo teste $t$, compararam-se os dados dos queijos tradicionais com os dados dos queijos acrescidos de EHS.

\section{3 - RESULTADOS E DISCUSSÃO}

\section{1 - Rendimento dos queijos}

Estão apresentadas nas Tabelas 1 e 2 as cifras de transição obtidas nos processamentos dos queijos Minas frescal com coalho bovino e com coagulante microbiano, e dos queijos com EHS processados com os mesmos agentes coagulantes, respectivamente, assim como o rendimento em gramas de sólidos totais por litro de 
leite (g ST/L) e, também, em litros de leite necessários para a produção de um quilograma de queijo (L/kg).

TABELA 1. Transição dos componentes do leite para os queijos tradicionais e rendimento dos processos nos tratamentos com coalho bovino e com coagulante microbiano

\begin{tabular}{lcc}
\hline Parâmetros & $\begin{array}{c}\text { Queijo Tradicional com } \\
\text { coalho bovino }\end{array}$ & $\begin{array}{c}\text { Queijo Tradicional com } \\
\text { coagulante microbiano }\end{array}$ \\
\hline Transição das proteínas (\%) & $72,33^{\mathrm{a}}$ & $73,00^{\mathrm{a}}$ \\
Transição da gordura (\%) & $85,33^{\mathrm{a}}$ & $85,33^{\mathrm{a}}$ \\
Transição de Sólidos Totais (\%) & $52,79^{\mathrm{a}}$ & $53,30^{\mathrm{a}}$ \\
Rendimento em g de ST/L & $65,87^{\mathrm{a}}$ & $67,17^{\mathrm{b}}$ \\
Rendimento (L/kg) & $6,09^{\mathrm{a}}$ & $6,00^{\mathrm{a}}$
\end{tabular}

$\mathrm{ST}=$ sólidos totais.

,b... médias com a mesma letra superescrita, na mesma linha, não são estatisticamente diferentes $(\mathrm{p}<0,05)$.

Não houve diferenças $(\mathrm{p}<0,05)$ na retenção de proteínas, gordura e sólidos totais, bem como no rendimento (L/kg), quando se comparou o queijo Minas frescal tradicional processado com coalho bovino e com coagulante microbiano (Tabela 1).

A cifra de transição de gordura do queijo Minas frescal processado com coalho bovino está bem abaixo do valor informado $(92,29 \%)$ no estudo desenvolvido por SABOYA et al. [26] que trabalharam com a mesma técnica de fabricação aqui realizada. Estes autores relataram uma menor perda da quantidade de gordura no soro (apenas 0,3 g/100mL) e o leite empregado apresentava $3,24 \mathrm{~g}$ de gordura/ $100 \mathrm{~mL}$, ao passo que no presente trabalho perdeu-se em média $0,52 \mathrm{~g}$ de gordura/ $100 \mathrm{~mL}$ e o leite utilizado nos processamentos apresentou menor quantidade deste nutriente (média de 3,00g/100mL). A presença de maior quantidade de gordura no soro encontrada no presente trabalho, também, pode ser explicada por diferenças que geralmente podem acontecer durante o corte e o tratamento da massa, duas etapas importantes para a fabricação de queijos, segundo FURTADO [10]. Neste experimento, o corte foi feito manualmente, não sendo possivel a padronização do tamanho dos grãos, causando provavelmente maior perda de gordura.

O rendimento em g de ST/L do queijo Minas frescal processado com coagulante microbiano foi $1,97 \%$ superior, em termos relativos, àquele processado com coalho bovino (Tabela 1), sendo estatisticamente significativo $(p<0,05)$. Não houve diferença $(p<0,05)$, entretanto, quando a comparação foi realizada com os valores obtidos para o rendimento em $\mathrm{L} / \mathrm{kg}$. Em termos relativos, contudo, a diferença é de $1,5 \%$, o que corresponde ao aumento preconizado pelo fabricante, a favor do coagulante microbiano [6], e isto pode resultar em beneficios econômicos numa produção em larga escala.

$\mathrm{O}$ rendimento em $\mathrm{g}$ de $\mathrm{ST} / \mathrm{L}$ encontrado por SABOYA et al. [26], também, foi superior $(67,86 \mathrm{~g} \mathrm{ST} / \mathrm{L})$ ao encontrado nesta pesquisa (Tabela 1), e isto se deve ao fato da maior transição de gordura do leite para o queijo conseguida pelos autores.
Os valores encontrados para os parâmetros dispostos na Tabela 1 não coincidem com os dados obtidos por ROSSI et al. [24], especialmente o rendimento, tanto em L/kg $(5,47)$ como em g de ST/L $(62,02)$, alcançado para o tratamento com coagulante microbiano. Estas diferenças podem estar relacionadas mais com as etapas de corte da massa e ao tratamento empregado na massa que ao tipo de agente coagulante. Além disto, as tecnologias de fabricação empregadas também apresentaram diferenças, já que os referidos autores utilizaram $2 \%$ de sal em relação ao volume de leite e adicionaram ácido lático em substituição à cultura lática, ao passo que neste trabalho a concentração de sal foi de $1,5 \%$ e a cultura lática foi empregada.

Comparando-se as cifras de transição entre os dois tipos de queijos com EHS (Tabela 2) nota-se que foi seguida a mesma tendência dos queijos tradicionais (Tabela 1), no entanto houve diferença $(\mathrm{p}<0,05)$ na transição da gordura além da diferença encontrada no rendimento em gramas de sólidos totais por litro (Tabela 2).

TABELA 2. Transição dos componentes da mistura (leite + EHS) para os queijos e rendimento dos processos nos tratamentos com coalho bovino e com coagulante microbiano

\begin{tabular}{lcc}
\hline Parâmetros & $\begin{array}{c}\text { Queijo com EHS e } \\
\text { coalho bovino }\end{array}$ & $\begin{array}{c}\text { Queijo com EHS } \\
\text { e coagulante } \\
\text { microbiano }\end{array}$ \\
\hline Transição das proteinas (\%) & $76,86^{\mathrm{a}}$ & $77,41^{\mathrm{a}}$ \\
Transição da gordura (\%) & $76,00^{\mathrm{a}}$ & $79,00^{\mathrm{b}}$ \\
Transição de Sólidos Totais (\%) & $54,17^{\mathrm{a}}$ & $54,83^{\mathrm{a}}$ \\
Rendimento em g de ST/L & $76,85^{\mathrm{a}}$ & $78,28^{\mathrm{b}}$ \\
Rendimento (L/kg) & $4,76^{\mathrm{a}}$ & $4,69^{\mathrm{a}}$ \\
\hline
\end{tabular}

$\mathrm{ST}=$ sólidos totais

médias com a mesma letra superescrita, na mesma linha não são estatisticamente diferentes $(\mathrm{p}<0,05)$.

A retenção de proteínas $(76,86$ e $77,41 \%)$ nos queijos adicionados de EHS foi maior $(\mathrm{p}<0,05)$ se comparada aos respectivos tradicionais $(72,33$ e $73,00 \%)$. Isto ocorreu em conseqüência da presença das proteínas de soja na massa dos queijos. O mesmo efeito se deu com a transição de sólidos totais dos queijos acrescidos de EHS (Tabela 2) pelo teste $t(p<0,05)$, que também pode ser explicado em virtude do maior teor protéico dos mesmos.

Houve, porém, menor transição da gordura $(76,00$ e $79,00 \%)$ para os queijos acrescidos de EHS. Verificou-se diferença $(p<0,05)$ entre os valores obtidos para a transição de gordura nos dois tratamentos com EHS (Tabela 2). Possivelmente, por causa da presença do derivado de soja, a estrutura da coalhada formada foi incapaz de aprisioná-la adequadamente na matriz protéica, e por isso sua perda no soro foi mais elevada, aproximadamente $0,90 \mathrm{~g} / 100 \mathrm{~mL}$, em relação aos soros obtidos durante o processamento dos queijos tradicionais $(0,58 \mathrm{e}$ $0,60 \mathrm{~g} / 100 \mathrm{~mL})$. A menor retenção de gordura em queijos 
contendo ingredientes de soja tem sido verificada por diversos autores $[11,16,17,18,23,27,28,31,32]$.

Outro parâmetro que apresentou diferença $(\mathrm{p}<0,05)$ entre os tratamentos com EHS foi o rendimento expresso em g de ST/L (Tabela 2) e, novamente, o queijo processado com coagulante microbiano indicou maior rendimento. O uso deste coagulante promoveu um aumento relativo de $1,86 \%$ no rendimento em relação ao seu análogo processado com coalho bovino. Este resultado pode ser explicado pela maior retenção de gordura na coalhada. Quando a comparação do rendimento foi feita com base na quantidade de litros de leite utilizada para processar um quilograma de queijo (L/kg), observou-se que foram necessários 4,76 e 4,69L para processar um quilograma de queijo com coalho bovino e com coagulante microbiano, respectivamente. Estes resultados, porém, não foram diferentes do ponto de vista estatístico $(\mathrm{p}<0,05)$.

Os rendimentos em litros de leite para processar um quilograma de queijo $(\mathrm{L} / \mathrm{kg}$ ) dos tratamentos com EHS produzidos com coalho bovino e com coagulante microbiano foram, em termos relativos, 27,94\% e 27,93\% superiores aos seus controles, respectivamente. Tendo em vista que o rendimento calculado g ST/L é um dado mais confiável porque leva em consideração o teor de sólidos totais do leite, estimou-se também a percentagem de aumento nestes tratamentos, sendo 16,67\% para o queijo com coalho bovino e $16,54 \%$ para o queijo com coagulante microbiano. Estes dados indicam que derivados de soja aumentam o rendimento desse tipo de queijo pelo fato de reterem maior teor de proteínas.

\section{2 - Estimativa do custo dos queijos}

A quantidade de queijo (kg/100L leite) obtida para cada tratamento, assim como o custo, em dólares americanos (US\$), estão indicados na Tabela 3.

Houve um aumento relativo no custo muito pequeno, igual a $0,68 \%$, e o rendimento em g ST/L foi $1,97 \%$ superior para o queijo com coagulante microbiano $(\mathrm{Ta}-$ bela 2). O queijo tradicional produzido com coagulante microbiano, portanto, tem mais vantagem em relação ao custo de processamento.

TABELA 3. Quantidade de queijo obtida (kg) e custo de cada formulação (US\$)

\begin{tabular}{lcc}
\hline Tratamentos & $\begin{array}{c}\text { Quantidade obtida para } \\
\text { 100L de leite }(\mathrm{kg})\end{array}$ & $\begin{array}{c}\text { Custo para 100L } \\
\text { de leite (US\$) }\end{array}$ \\
\hline $\begin{array}{l}\text { Queijo tradicional com coalho bovino } \\
\text { Queijo tradicional com coagulante }\end{array}$ & $16,41^{\mathrm{a}}$ & 35,12 \\
microbiano & $16,67^{\mathrm{a}}$ & 35,36 \\
Queijo com EHS e com coalho bovino & $21,19^{\mathrm{b}}$ & 36,75 \\
$\begin{array}{l}\text { Queijo com EHS e com coagulante } \\
\text { microbiano }\end{array}$ & $22,21^{\mathrm{b}}$ & 37,50 \\
\hline
\end{tabular}

b.... médias com a mesma letra superescrita, na mesma coluna não são estatisticamente diferentes $(\mathrm{p}<0,05)$.

Como já citado na metodologia, a única diferença que houve no desenvolvimento da formulação-base alternativa foi a substituição do coalho bovino pelo coagulante microbiano, portanto o custo desta formulação ficou em US\$ 37,50. Comparando os custos das formulações-base empregando-se 100L de leite, encontrou-se uma diferença de US\$ 0,75, o que representa um aumento relativo de 2,04\%. Como aconteceu com os queijos Minas frescal tradicionais, o rendimento da formulação-base processada com coagulante também foi superior $(1,86 \%)$, comprovando mais uma vez que apesar do custo do coagulante ser bem superior (US\$ 32,00 a 35,00/L) ao coalho bovino (US\$ 3,00/kg), não há comprometimento no custo.

Estabelecendo-se um paralelo entre o custo dos queijos tradicionais com coalho bovino e com coagulante microbiano, e dos queijos com adição de EHS com os mesmos agentes coagulantes, estimou-se um custo adicional aos últimos queijos citados igual a 4,64 e $6,05 \%$, respectivamente.

O aumento do custo de cada formulação em relação aos tradicionais deve-se à introdução de EHS no processamento e aos teores mais elevados de agentes coagulantes e cloreto de cálcio. Deve-se considerar, contudo, que essas formulações-base apresentaram rendimento adicional - em g ST/L - ao redor de 16,5\%. Além disto, o preço final ao consumidor poderia ser maior, uma vez que produtos com menor teor de gordura, conseqüentemente, menos calorias, podem ser comercializados como produtos light e apresentam preços mais elevados que os tradicionais. O uso de EHS, portanto, agregaria valor ao queijo final.

\section{4 - CONCLUSÕES}

A inclusão do extrato hidrossolúvel de soja desengordurado (EHS) em queijo tipo Minas frescal promove maior transição de proteínas e sólidos totais e diminui a retenção da gordura. O queijo processado com EHS e com coalho bovino foi $16,67 \%$ superior no rendimento (g ST/L) em relação ao queijo tradicional processado com o mesmo coalho. O mesmo ocorreu com o coagulante microbiano com rendimento de 16,54\%.

O custo das formulações-base (100kg queijo) processadas com coalho bovino e com coagulante microbiano foi US\$ 36,75 e US\$ 37,50 , respectivamente, ao passo que para os queijos tradicionais os custos foram equivalentes a US\$ 35, 12 e US\$ 35,36, respectivamente.

\section{5 - REFERÊNCIAS BIBLIOGRÁFICAS}

[1] ASSOCIAÇÃO BRASILEIRA DAS INDÚSTRIAS DE QUEIJO. Informações sobre produção de queijo tipo Minas frescal no Brasil. Disponível em: <http:// www.abiq.com.br>. Acesso em: 13 mar. 2003.

[2] ASSOCIATION OFFICIAL ANALYSIS CHEMISTRY. Official methods of analysis of AOAC international: food composition; additives, natural contaminants. $16^{\text {th }}$. ed. Virginia: Association Analytical Chemists, 1995. v. 2. cap. 33, p. 1-75.

[3] BARBANO, D. H; RASMUSSEN, R. R. Cheese yield performance of fermentation-produced chymosin and other milk coagulants. J. Dairy Sci., Champaign, v. 75, n. 1, p. 1-2, Jan. 1992. 
[4] BONASSI, I. A.; GOLDONI, J. S.; KROLL, L. B. Influência das bactérias láticas mesófilas: Streptococcus cremoris, Streptococcus lactis, Streptococcus diacetilactis e Leuconostoc citrovorum nas características do queijo Minas: propriedades organolépticas. Ciênc. Tecnol. Aliment., Campinas, v. 3, n. 1, p. 24-34, jan. 1983.

[5] BRASIL. Ministério da Agricultura, Pecuária e Abastecimento. Produção e industrialização de produtos lácteos em 2000. Disponível em: <www.agricultura.gov.br/htm1/contas / 09dfamt2001.htm>. Acesso em: 12 jan. 2003.

[6] CHY-MAX, o novo coalho da Chr.-Hansen que proporciona aumento no rendimento. Disponivel em: <http;// www.milkenet.com.Br/hala/02.html>. Acesso em: 22 out. 2003.

[7] CUNHA, C. R. et al. Efeito do fator concentração o rendimento do queijo Minas frescal de baixo teor de gordura fabricado por ultrafiltração. Ciênc. Tecnol. Aliment., Campinas, v. 22, n. 1, p. 76-81, jan./abr. 2002.

[8] FOX. P. F.; LAW, J. Enzimology of cheese ripening. Food Biotech., v. 5, n. 3, p. 239-262, 1991.

[9] FOX. P. F. Advanced dairy chemistry: proteins. London: Blackie Academic \& Professional, 1997. v. 1.

[10] FURTADO, M. M. A arte e a ciência do queijo. São Paulo: Globo, 1990.

[11] FURTADO, M. M.; COElho, D. F.; GOMES, J. C. Influência do tratamento da farinha de soja integral précozida para produção do queijo Mussarela. Rev. ILCT, Juiz de Fora, v. 43, n. 259, p. 25-32, 1988.

[12] FURTADO, M. M.; LOURENÇO-NETO, J. P. M. Tecnologia de queijos: manual técnico para produção industrial de queijos. São Paulo: Dimepar, 1994.

[13] FURTADO, M. M.; WOLFSCHOON-POMBO, A. F. Fabricação de queijo Prato e Minas: estudo do rendimento - determinação das cifras de transição. Rev. ILCT, Juiz de Fora, v. 34, p. 3-19, set./out. 1979a.

[14] . Fabricação de queijo Prato e Minas: estudo do rendimento - previsão de gordura no extrato seco. Rev. ILCT, Juiz de Fora, v. 34, p. 3-11, nov./dez. 1979b.

[15] FURTADO, M. M. O rendimento da fabricação de queijos: métodos para avaliação e comparação. In: SEMINÁRIO INTERNACIONAL DE QUEIJOS FRESCOS, 1. 1998, Atibaia.. Anais. Atibaia: CHR HANSEN/HAHN/ GEA/EQUITEC/ TECLAB, 1998. p. 78-94.

[16] FURTADO, M. M.; COElho, D. F.; GOMES, J. C. Influência do tratamento da farinha de soja integral précozida para produção do queijo Mussarela. Rev. ILCT, Juiz de Fora, v. 43, n. 259, p. 25-32, 1988.

[17] _. Estudo do rendimento e das características físico-químicas e sensoriais de queijo Prato elaborado com leite adicionado de farinha de soja integral précozida. Arq. Biol. Tecnol., Curitiba, v. 32, n. 4, p. 759-773, out. 1989.

[18] GOMES, J. C. et al. Substituição parcial do leite por farinha de soja em queijo Prato: características químicas. Arq. Biol. Tecnol., Curitiba, v. 30, n. 4, p. 615632, nov. 1987.

[19] LOURENÇO-NETO, J. P. de M. O uso de culturas láticas na fabricação de Minas frescal como alternativa de melhoria de qualidade. In: SEMINÁRIO INTERNACIONAL "QUEIJOS FRESCOS", 1, Atibaia, 1998. Trabalho apresentado.

[20] LOURENÇO-NETO, J. P. de M.; SOBRAL, M. L. Uso de culturas termófilas na fabricação de queijo minas frescal. In: CONGRESSO NACIONAL DE LATICÍNIOS, 9. Juiz de Fora, 1986. Anais. Juiz de Fora, 1986.
[21] MEDINA, M. et al. Characteristics of Burgos and Hispanic cheeses manufactured with calf rennet or with recombinant chymosin. Food Chem., Oxford, v. 45, p. 85-89, 1992.

[22] MELLO, F. D. Sistema Militza - M II: uma nova alternativa para a fabricação de Minas frescal. In: SEMINÁRIO INTERNACIONAL DE QUEIJOS FRESCOS, 1., 1998, Atibaia. Anais. Atibaia: CHR HANSEN/HAHN/ GEA/EQUITEC/TECLAB, 1998. p. 26-32.

[23] NEVES-SOUZA, R. D. D. Queijo tipo Minas frescal com isoflavonas: abordagem físico-química, textural, sensorial e microbiológica. Londrina, 2003, $166 \mathrm{f}$. Tese (Doutorado em Ciência de Alimentos) - Universidade Estadual de Londrina., Londrina, 2003.

[24] ROSSI, D. A. et al. Utilização do coalho bovino e coagulantes microbiano e genético na composição e rendimento do queijo Minas frescal. Rev. ICLC, Juiz de Fora, v. 53, n. 305, p. 8-14, set./dez. 1998a.

[25] ROSSI, D. A. et al. Utilização do coalho bovino e coagulantes microbiano e genético na proteólise e durabilidade do queijo Minas frescal. Rev. ICLC, Juiz de Fora, v. 53, n. 302/303, p. 75-84, jan./jun. 1998b.

[26] SABOYA, L. V. et al. Efeitos físico-químicos da adição de leite reconstituído na fabricação de queijo Minas frescal. Ciênc. Tecnol. Aliment., Campinas, v. 18, n. 4, p. 368-376, out./dez. 1998.

[27] SOUZA, R. D. N. Utilização do extrato de soja desengordurado em pó na produção de queijo Minas frescal: desenvolvimento tecnológico e caracterização do produto. Londrina, 1992, 114 p. Dissertação (Mestrado em Ciência de Alimentos) - Universidade Estadual de Londrina.

[28] SOUZA, R. D. N.; ANTUNES, L. A. F.; DA SILVA, R. S. F. Produção de queijo Minas frescal contendo proteína de soja. Ciênc. Tecnol. Aliment., Campinas, v. 12, n. 2, p. 145-152, jul./dez. 1992.

[29] VAN DENDER, A. G. F.; MORENO, I. Estudo de processos alternativos para a fabricação de queijo Minas frescal. Rev. ILCT, Juiz de Fora, v. 47, n. 279/281, p. 76-77, 1992.

[30] VAN DENDER, A. G. F. Contribuição ao estudo do uso de ultrafiltração na fabricação de queijo Minas frescal. Campinas, 1995, $176 \mathrm{f}$. Tese (Doutorado em Ciência de Alimentos) - Faculdade de Engenharia de Alimentos, Universidade Estadual de Campinas, Campinas.

[31] VENKATACHALAM, N.; REDDY, M. I.; McMAHON, D. J. Effect of soy protein isolate on rennet coagulation of milk and microstructure of cheese curd. In: IFT ANNUAL MEETING, Logan, 1995. Trabalho apresentado.

[32] VIANA, A. M. L. et al. Utilização de extrato solúvel de soja e farinha de soja integral pré-cozida na produção de queijo Prato e queijo Minas frescal. Rev. ILCT, Juiz de Fora, v. 42, n. 253, p. 9-15, 1987.

[33] VIEIRA, S. D. A. et al. Éléments de fabrication d'un nouveau fromage brésilien de type "Minas frescal" par le procédé M.M.V. La Technique Laitière, Rennes, n. 978, p. 17-20, juil./août. 1983.

[34] Parâmetros para a fabricação de queijo Minas frescal por meio de ultrafiltração. Rev. ILCT, Juiz de Fora, v. 39, n. 235, p. 53-58, 1984.

\section{6 - AGRADECIMENTOS}

Os autores agradecem ao Prof. Dr. Lúcio Alberto Forti Antunes, da Chr. Hansen do Brasil, pelas valiosas sugestões apresentadas durante a execução desta pesquisa. 